\title{
Analysis of Genetic Variation in CD40 and CD40L: Relationship with mRNA Relative Expression and Soluble Proteins in Acute Coronary Syndrome
}

\author{
D. E. Martínez-Fernández, ${ }^{1,2}$ J. R. Padilla-Gutiérrez $\mathbb{D}^{1}{ }^{1}$ F. Casillas-Muñoz, ${ }^{1,3}$ \\ Emmanuel Valdés-Alvarado (D, ${ }^{1}$ Brenda Parra-Reyna, ${ }^{1,3}$ Maricela Aceves-Ramírez, ${ }^{1,3}$ \\ J. F. Muñoz-Valle $\mathbb{D}^{1},{ }^{1}$ U. Zalapa Flores, ${ }^{4}$ J. C. Chávez Herrera, ${ }^{4}$ and Y. Valle ${ }^{1}{ }^{1}$ \\ ${ }^{1}$ Instituto de Investigación en Ciencias Biomédicas (IICB), Centro Universitario de Ciencias de la Salud (CUCS), Universidad de \\ Guadalajara (UdeG), Sierra Mojada \#950, Colonia Independencia, C.P, 44340 Guadalajara, Jalisco, Mexico \\ ${ }^{2}$ Doctorado en Ciencias Biomédicas, Centro Universitario de Ciencias de la Salud (CUCS), Universidad de Guadalajara (UdeG), \\ Guadalajara, Jalisco, Mexico \\ ${ }^{3}$ Doctorado en Genética Humana, Centro Universitario de Ciencias de la Salud (CUCS), Universidad de Guadalajara (UdeG), \\ Guadalajara, Jalisco, Mexico \\ ${ }^{4}$ Especialidad en Cardiología, Unidad Médica de Alta Especialidad, Centro Médico Nacional de Occidente (CMNO), \\ Departamento de Cardiología, Instituto Mexicano del Seguro Social (IMSS), Guadalajara, Jalisco, Mexico
}

Correspondence should be addressed to Y. Valle; yemivalle@yahoo.com.mx

Received 17 December 2018; Revised 13 March 2019; Accepted 10 April 2019; Published 30 April 2019

Academic Editor: Cinzia Ciccacci

Copyright (c) 2019 D. E. Martínez-Fernández et al. This is an open access article distributed under the Creative Commons Attribution License, which permits unrestricted use, distribution, and reproduction in any medium, provided the original work is properly cited.

\begin{abstract}
Acute coronary syndrome (ACS) can be triggered by the presence of inflammatory factors which promote the activation of immune cells by costimulatory molecules such as CD 40 and its ligand CD40L. Environmental and genetic factors are involved in the etiology of the ACS. The aim of this study was to explore the gene and protein expression associated with CD40 and CD40L genetic variants in ACS patients from the western Mexican population. A total of 620 individuals from western Mexico were recruited: 320 ACS patients and 300 individuals without a history of ischemic cardiopathy were evaluated. The genotype was determined using TaqMan SNP genotyping assays. CD40 and CD40L expressions at the mRNA level were quantified using TaqMan Gene Expression Assays. Soluble protein isoforms were measured by enzyme-linked immunosorbent assay. We did not find evidence of association between CD40 (rs1883832, rs4810485, and rs11086998) and CD40L (rs3092952 and rs3092920) genetic variants and susceptibility to ACS, although rs1883832 and rs4810485 were significantly associated with high sCD40 plasma levels. Plasma levels of sCD40L can be affected by gender and the clinical spectrum of acute coronary syndrome. Our results do not suggest a functional role of $C D 40$ and CD40L genetic variants in ACS. However, they could reflect the inflammatory process and platelet activation in ACS patients, even when they are under pharmacological therapy. Due to the important roles of the CD40-CD40L system in the pathogenesis of ACS, longitudinal studies are required to determine if soluble levels of CD40 and CD40L could be clinically useful markers of a recurrent cardiovascular event after an ACS.
\end{abstract}

\section{Introduction}

Cardiovascular disease is the leading cause of death worldwide [1], specifically acute coronary syndrome (ACS) characterized by the acute reduction in coronary blood flow and myocardial ischemia or necrosis. Three clinical entities are distinguished: ST-segment elevation myocardial infarction (STEMI), non-ST segment elevation myocardial infarction (NSTEMI), and unstable angina (UA), with a common etiology; however, they may have different outcomes [2]. For this 
reason, the early prognosis and risk stratification are the first line of action to reduce the risk of recurrent episodes or even death after an ACS [3].

In the etiology of ACS, metabolic, genetic, environmental, and among other factors are involved, conditions that can increase inflammation and endothelial dysfunction, key elements in the development of atherosclerosis [4, 5]. It has been estimated that the percentage of heritability is 40 $55 \%$ of the interindividual variation in the risk of ACS supporting the substantial genetic influence [6].

CD40 is a costimulatory molecule that is expressed constitutively in B lymphocytes and expressed in other cells such as monocytes, macrophages, endothelial cells (ECs), and smooth muscle cells (SMCs) [7]. Its ligand, CD40L, is expressed mainly on the surface of platelets (95\%), $\mathrm{T}$ lymphocytes, ECs, SMCs, and macrophages [7, 8].

The interaction of CD40 and CD40L is involved in the immunopathogenesis of ACS [9] due to its participation in bidirectional cell activation through the signaling pathways c-Jun, NF- $\kappa$ B, and ERK $1 / 2$, promoting the increase of adhesion molecules, secretion of proinflammatory cytokines, and platelet activation [7]. However, the soluble forms of CD40 and CD40L have been associated with endothelial dysfunction or as markers of adverse cardiovascular events in ACS $[10,11]$ patients suggesting them as potential targets of therapeutic agents [9].

In this regard, single-nucleotide polymorphisms (SNPs) have been identified in the CD40 and CD40L genes that may increase the susceptibility for the development of ACS, through genome-wide association studies [7] and candidate gene studies [12-16]. These genetic variants have been associated with inflammatory and autoimmune diseases $[17,18]$, with alterations in the CD40 and CD40L mRNA expression [19-25] as well as the regulation of soluble forms modifying their function $[15,20]$ which have an effect in the initiation, development, and progression of atherosclerosis [26, 27].

Inflammation and endothelial dysfunction are factors that promote the development of atherosclerosis; however, the underlying cellular and molecular interactions, which link the existing risk factors with the atherosclerotic process, are not well characterized. To our knowledge, there are no studies that evaluate the contribution of CD40 and CD40L polymorphisms in the population from western Mexico associated with cardiovascular diseases specifically with ACS.

Accordingly, the aim of this study was to explore the association between single-nucleotide polymorphisms in the $C D 40$ (rs1883832 (c.-1C>T), rs4810485 (c.51+914G>T), and rs11086998 (c.679C>G)) and CD40L (rs3092952 (g.1615A>G) and rs3092920 (18656G>T)) genes and susceptibility to ACS. We also explore the association between these polymorphisms with CD40 and CD40L expression in peripheral blood leukocytes and soluble levels quantified in plasma samples obtained from ACS patients and subjects without ischemic cardiopathy included in a control group (CG).

\section{Materials and Methods}

2.1. ACS Patients and Subjects in the Control Group. The study cohort consisted of 320 patients diagnosed with ACS classified according to the American College of Cardiology [28] and 300 subjects with ACS risk factors but no history of ischemic cardiopathy (ascertained by questionnaire) as the CG. All the individuals included in the study from western Mexico were recruited from Hospital de Especialidades del Centro Medico Nacional de Occidente del Instituto Mexicano del Seguro Social (CMNO-IMSS). The period of the study was from February 2016 to June 2018.

2.2. Ethical Considerations. The study was conducted in accordance with the 2013 Helsinki Declaration. All individuals agreed to participate in the study and signed informed written consent. The ethical approval was obtained by the Centro Universitario de Ciencias de La Salud (CUCS), UdeG (CI/065/2014).

2.3. Genotyping and Quality Control. Genetic variants were genotyping using TaqMan Assays for CD40 (rs1883832 (C_11655919_20), rs4810485 (C__1260190_10), and rs11086998 (C__1260325_10)) and for CD40L (rs3092952 (C_26154899_10) and rs3092920 (C_27452204_10)) using TaqMan Genotyping Master Mix (Applied Biosystems, Foster City, CA, USA), following the manufacturer's protocol. As quality control, a double-blind genotyping of $25 \%$ of the samples was performed for all polymorphisms, with no variation in the genotype assignment.

2.4. Real-Time PCR Analysis. Total RNA was extracted from peripheral blood leukocytes using TRIzol reagent (Invitrogen, Carlsbad, CA, USA) according to the manufacturer's instruction to obtain total RNA according to the method of Chomczynski and Sacchi [29]. One microgram of total RNA was reverse transcribed using reverse transcription reagents to obtain cDNA following the manufacturer's protocol (Promega Corporation, USA). Real-time PCR was performed using TaqMan probes: glyceraldehyde 3-phosphate dehydrogenase (GAPDH) (Hs02786624_g1), CD40 (Hs01002915_ g1), and CD40L (Hs00163934_m1) according to the conditions indicated in TaqMan Gene Expression Assay Protocol (Applied Biosystems, Foster City, CA, USA) using a Roche LightCycler $96^{\circledR}$ System. Relative expression was normalized to the expression level using GAPDH as an internal control and was evaluated using the $2^{-\Delta \Delta \mathrm{Cq}}$ and $2^{-\Delta \mathrm{Cq}}$ methods [30]. Results are expressed as a relative fold increase compared with control and unit relative of expression (URE), respectively. The $2^{-\Delta \Delta \mathrm{Cq}}$ method was calculated as is suggested by Livak and Schmittgen [30]; the aim of this method is to compare the expression measures of genes of interests through the normalization to a reference gene in this case GAPDH. Using the following equation: $\Delta \mathrm{Cq}=$ (average $\mathrm{Cq}$ of the gene of interest - average $\mathrm{Cq}$ of reference gene), we calculated $\Delta \Delta \mathrm{Cq}=(\Delta \mathrm{Cq}-\Delta \mathrm{Cq}$ calibrator $)$, where $\Delta \mathrm{Cq}$ calibrator refers to the average $\Delta \mathrm{Cq}$ of the control group. We determined the relative CD40 mRNA expression in peripheral blood leukocytes from ACS patients $(n=42)$ and control group $(n=18)$. For the assessment of the expression levels, cohort characteristics were as follows: age $(62 \pm 8)$, gender (female/male ratio; ACS: 1.7, CG: 1.6), and similar risk factor presence, and the same number of patients was included for 
each of the three clinical entities of ACS (14 UA, 14 NSTEMI, and 14 STEMI).

2.5. Detection of $s C D 40, s C D 40 L$, and IFN- $\gamma$. The levels of sCD40, sCD40L, and IFN- $\gamma$ were determined on plasma samples using enzyme-linked immunosorbent assays (ELISA) (Human CD40 Quantikine ELISA Kit, Human CD40 Ligand Quantikine, and Human IFN-gamma Quantikine ELISA Kit, R\&D Systems) according to the manufacturer's protocol. The assay sensitivity for $s \mathrm{CD} 40, \mathrm{sCD} 40 \mathrm{~L}$, and IFN $-\gamma$ was $5.56 \mathrm{pg} / \mathrm{mL}, 10.1 \mathrm{pg} / \mathrm{mL}$, and $8 \mathrm{pg} / \mathrm{mL}$, respectively.

2.6. Statistical Analysis. Statistical analysis was performed using SPSS software version 22 (SPSS Inc., Chicago, IL, USA). Data were presented as a median and interquartile range (IQR) unless otherwise indicated. The Mann-Whitney $U$ test and Kruskal-Wallis test were used to compare differences between groups. Genotype and allele frequencies of CD40 and CD40L genetic variants were compared between the ACS patients and control group. Odds ratios (OR) and 95\% confidence intervals (CIs) were calculated using Chi-squared and Fisher's exact tests when appropriate. Bonferroni's correction was applied to reduce the statistical type 1 error $(\mathrm{pc})$ in multiple comparisons. Hardy-Weinberg equilibrium was calculated from genotype distribution, and the linkage disequilibrium (LD) between the polymorphisms was calculated using Lewontin's $D^{\prime}$ and $r^{2}$ between genetic markers. The haplotypes and their frequencies were estimated using the SHEsis software platform (http://analysis .bio-x.cn/myAnalysis.php). A multilinear regression model was used to estimate the association of common risk factors (comorbidities, gender, medication intake, etc.) with sCD40, sCD40L, and mRNA expression levels. Correlation analysis was ascertained by Spearman's correlation.

\section{Results}

3.1. Clinical Features. The clinical information of the 320 ACS patients and 300 control subjects was recorded in Table 1. ACS patients and CG subjects had a mean age of 62 ( \pm 11 standard deviation (SD)) and $55( \pm 10 \mathrm{SD})$ years, respectively. In the ACS group, there are 3.32 more males than females; while in the CG, the male/females ratio was almost $1 / 1$. ACS patients presented high levels of cardiac function markers including $\mathrm{CK}, \mathrm{CK}-\mathrm{MB}$, and troponin I with respect to the reference values; the biochemical parameters are significantly elevated in ACS patients compared to control as shown in Table 1. The risk factors with the highest prevalence in ACS were high blood pressure, smoking, and diabetes mellitus type 2. Reinfarction was found in 49 cases (15.3\%). The medication intake included antiplatelet (acetylsalicylic acid, clopidogrel), statins, anticoagulants (heparin and enoxaparin), and antihypertensive agents (captopril, enalapril, spironolactone, and furosemide).

3.2. Genotype and Allele Analysis. The genotype and allele frequencies of the CD40 (rs1883832, rs4810485, and rs11086998) and CD40L (rs3092952 and rs3092920) polymorphisms are shown in Tables 2-4. Genotype distributions agreed with Hardy-Weinberg equilibrium expectations $(p>0.05)$ for all SNPs.

Neither genotype nor allele distribution of three CD40 polymorphisms was statistically different when the ACS and control groups were compared.

Because CD40L is located on the $\mathrm{X}$ chromosome, we performed the analysis by gender (Table 3). It is noteworthy to mention that we did not find differences for allele frequencies on CD40L variants between genders (rs3092952 A/G: $p=0.73 ;$ rs3092920 G/T: $p=0.96$ ). Genetic distribution of both polymorphisms was similar between patients and controls either pooled (data not shown) or gender separated.

3.3. Haplotype Analysis of CD40 and CD40L. We analyzed the association between haplotypes found in our western Mexican cohort of the CD40/CD40L genes and ACS. Analysis showed that rs1883832, rs4810485, and rs11086998 of $C D 40$ were in linkage disequilibrium $\left(D^{\prime}=0.83, r^{2}=0.021\right.$, $p=0.010)$. We found one major haplotype (CCG) that accounted for $67.1 \%$ and $65.5 \%$ in ACS patients and control subjects, respectively. We also found a protective haplotype (CTC, OR: $0.22 ; p=0.0001$ ) (Table 4).

CD40L polymorphisms were not in $\operatorname{LD}(p=0.756)$, indicating 3092952 and rs3092920 need to be analyzed separately.

3.4. CD40 mRNA Expression. ACS patients exhibited higher median expression of CD40 mRNA in peripheral blood leukocytes compared to controls ( 0.82 -fold more); however, this comparison was not significant. Similarly, stratification by clinical diagnosis in the ACS group (UA, NSTEMI, and STEMI) indicated no differences in the level of mRNA expression of $C D 40$. Next, we analyzed the data from the ACS patients and CG stratifying the results by carrying rs1883832, rs4810485, and rs11086998 genotypes. Because of the low frequencies of minor alleles, we assumed the dominant model of association (rs1883832: $\mathrm{C} / \mathrm{C}$ vs. $\mathrm{C} / \mathrm{T}+$ TT, r4810485: G/G vs. G/T+TT, and rs11086998: C/C vs. $\mathrm{C} / \mathrm{G}+\mathrm{G} / \mathrm{G})$, although we did not find differences in the level of $C D 40$ expression between genetic variants in either group (data not shown).

3.5. CD40L mRNA Expression. Analysis by the $2^{-\Delta \Delta \mathrm{Cq}}$ method showed that CD40L mRNA expression in ACS patients was 1.25-fold higher than that in the CG (Figure 1(a)); this difference was significantly ascertained by the $2^{-\Delta \mathrm{Cq}}$ method $(p=0.035)$ (Figure 1(b)). By clinical entities, we did not find any difference (data not shown).

Given the location on the X chromosome and the conclusion of other studies that CD4OL mRNA expression can be affected by gender [20], we analyzed separately for each gender in both groups of study without any evidence of association. By gender, either female or male patients tended to show more expression compared to the control group (Figure 2(a)). When we stratified by clinical diagnosis of ACS and gender, UA female patients showed the highest expression (5.12 URE), although none of the comparisons were statistically significant (male patients, $p=0.93$; female patients, $p=0.53$ ) (Figure 2(b)). 
TABLE 1: Clinical and demographic features of the CG and ACS patients.

(a)

\begin{tabular}{|c|c|c|c|c|}
\hline Variable & ACS median (IQR 25-75) & CG median (IQR 25-75) & Reference values & $p$ \\
\hline Age (years) & $62 \pm 11^{\mathrm{a}}$ & $55 \pm 10^{\mathrm{a}}$ & - & $<0.001$ \\
\hline Male/female ratio & 3.32 & 1.05 & - & $<0.001$ \\
\hline Cholesterol total (mg/dL) & $115(93-139)$ & $162(138-197)$ & $150-199$ & $<0.001$ \\
\hline Glucose (mg/dL) & $125(97-171)$ & $94(82-118)$ & $75-105$ & $<0.001$ \\
\hline Triglycerides (mg/dL) & $89(72-108)$ & $99(80-133)$ & $<200$ & $<0.001$ \\
\hline LDL-c (mg/dL) & $40(33-52)$ & $68(51-95)$ & $<130$ & $<0.001$ \\
\hline HDL-c (mg/dL) & $19(13-26)$ & $37(23-54)$ & $>40$ & $<0.001$ \\
\hline $\mathrm{CRP}(\mathrm{mg} / \mathrm{L})$ & $19(3.3-34.9)$ & $9.4(8.2-11.8)$ & $1-10^{*}$ & $<0.001$ \\
\hline Apo A-I (mg/dL) & $166(148-184)$ & $197(179-213)$ & $94-178$ & $<0.001$ \\
\hline Apo B (mg/dL) & $132(109-155)$ & $166(147-186)$ & $63-133$ & $<0.001$ \\
\hline $\mathrm{CK}(\mathrm{IU} / \mathrm{mL})$ & $361(147-1023)$ & N.A. & 24-195 & - \\
\hline CK-MB (IU/mL) & $48(22-131)$ & N.A. & $<12$ & - \\
\hline Troponin I (ng/mL) & $3(0.57-7.1)$ & N.A. & $0.1-0.4$ & - \\
\hline
\end{tabular}

(b)

\begin{tabular}{lccr}
\hline Risk factor & ACS $n(\%)$ & CG $n(\%)$ & $p$ \\
\hline Obesity & $87(27.2)$ & $35(11.1)$ & $<0.001$ \\
Diabetes mellitus type 2 & $148(46.3)$ & $59(18.7)$ & $<0.001$ \\
Dyslipidemia & $140(43.8)$ & $45(14.2)$ & $<0.001$ \\
High blood pressure & $214(66.9)$ & $90(28.5)$ & $<0.001$ \\
Smoking & $159(49.7)$ & $43(13.6)$ & $<0.001$ \\
\hline
\end{tabular}

(c)

\begin{tabular}{lccc}
\hline ACS diagnosis & $n(\%)$ & ACS treatment & $n(\%)$ \\
\hline UA & $38(11.9)$ & Acetyl salicylic acid & $304(95)$ \\
NSTEMI & $64(20)$ & Statins & $291(91)$ \\
STEMI & $218(68.1)$ & Antiplaquetary agents & $289(90.3)$ \\
& & Antihypertensive agents & $180(56.2)$ \\
& & Anticoagulants agents & 219 $(68.3)$ \\
& & Beta-blockers & 181 (56.6) \\
& & Nitrates (isosorbide) & $69(21.7)$ \\
\hline
\end{tabular}

${ }^{*}$ Mann-Whitney $U$ test; ACS: acute coronary syndrome; Apo A-I: apolipoprotein A-I; Apo B: apolipoprotein B; CG: control group; CK: creatinine kinase; CK-MB: creatinine kinase muscle and brain; CRP: C-reactive protein; HDL-c: high-density lipoprotein; IQR: interquartile range; LDL-c: low-density lipoprotein; NSTEMI: non-ST-segment elevation myocardial infarction; N.A.: not applicable; UA: unstable angina; STEMI: ST-segment elevation myocardial infarction (STEMI). The data were expressed as a median and interquartile range (Q25-Q75) unless otherwise indicated. ${ }^{\mathrm{a}}$ Data provided in mean \pm SD. ${ }^{*}$ Age-dependent.

It is important to mention that we explored the CD40L $m R N A$ expression in both groups of study according to genetic variants (rs3092952 and rs3092920), following the dominant model; however, we did not find differences.

3.6. Association between sCD40 and sCD40L with ACS. We evaluated plasma levels of CD40 and CD40L in both study groups. We found that ACS patients present significantly higher levels of sCD40 $(p>0.001)$ compared with the control group (843.3 vs. $492 \mathrm{pg} / \mathrm{mL}$ ). A multilinear regression model denotes that this association could be masked by clopidogrel $(p=0.005)$. ACS diagnosis seems not to be associated with sCD40 (UA: $677.1 \mathrm{pg} / \mathrm{mL}$; NSTEMI: $661.7 \mathrm{pg} / \mathrm{mL}$; and STEMI: $782.4 \mathrm{pg} / \mathrm{mL}, p=0.44)$.

Differences between sCD40 levels among CD40 genetic variants were also analyzed assuming the dominant model of association. As shown in Figure 3, C/C carriers had higher plasma sCD40 levels than rs1883832 C/T+T/T (561 vs. $475.7 \mathrm{pg} / \mathrm{mL}, p=0.005$ ). Similarly, homozygous carriers of rs4810485 showed a higher concentration of soluble CD40 than G/T+T/T carriers (562.5 vs. $489.7 \mathrm{pg} / \mathrm{mL}$, $p=0.015$ ). 
TABLE 2: Allele and genotype frequencies of the CD40 rs1883832, rs4810485, and rs11086998 in the ACS and CG.

\begin{tabular}{|c|c|c|c|c|}
\hline & $\begin{array}{c}\text { ACS } \\
n(\%)\end{array}$ & $\begin{array}{c}\text { CG } \\
n(\%)\end{array}$ & OR (CI 95\%) & $p$ \\
\hline \multicolumn{5}{|c|}{ rs1883832 } \\
\hline \multicolumn{5}{|c|}{ Genotype } \\
\hline $\mathrm{C} / \mathrm{C}$ & $188(58.8)$ & $194(64.7)$ & 1 & - \\
\hline $\mathrm{C} / \mathrm{T}$ & $121(37.8)$ & $95(31.7)$ & $1.31(0.94-1.84)$ & 0.10 \\
\hline $\mathrm{T} / \mathrm{T}$ & $11(3.4)$ & $11(3.6)$ & $1.03(0.44-2.44)$ & 0.94 \\
\hline \multicolumn{5}{|c|}{ Allele } \\
\hline $\mathrm{C}$ & $497(77.7)$ & $483(80.5)$ & \multirow{2}{*}{$1.19(0.90-1.56)$} & \multirow{2}{*}{0.22} \\
\hline $\mathrm{T}$ & $143(22.3)$ & $117(19.5)$ & & \\
\hline \multicolumn{5}{|c|}{ rs4810485 } \\
\hline \multicolumn{5}{|c|}{ Genotype } \\
\hline G/G & $186(58.1)$ & $173(57.6)$ & 1 & - \\
\hline $\mathrm{G} / \mathrm{T}$ & $118(36.9)$ & $113(37.7)$ & $0.97(0.70-1.35)$ & 0.86 \\
\hline $\mathrm{T} / \mathrm{T}$ & $16(5)$ & $14(4.7)$ & $1.06(0.50-2.24)$ & 0.87 \\
\hline \multicolumn{5}{|c|}{ Allele } \\
\hline G & $490(76.6)$ & $459(76.5)$ & \multirow{2}{*}{$0.99(0.77-1.30)$} & \multirow{2}{*}{0.97} \\
\hline $\mathrm{T}$ & $150(23.4)$ & $141(23.5)$ & & \\
\hline \multicolumn{5}{|c|}{ rs11086998 } \\
\hline \multicolumn{5}{|c|}{ Genotype } \\
\hline $\mathrm{C} / \mathrm{C}$ & $268(83.8)$ & $242(80.7)$ & 1 & - \\
\hline $\mathrm{C} / \mathrm{G}$ & $47(14.7)$ & $52(17.3)$ & $0.82(0.53-1.26)$ & 0.36 \\
\hline G/G & $5(1.5)$ & $6(2)$ & $0.75(0.23-2.50)$ & 0.64 \\
\hline \multicolumn{5}{|c|}{ Allele } \\
\hline $\mathrm{C}$ & $583(91)$ & $536(89.3)$ & \multirow{2}{*}{$0.82(0.56-1.19)$} & \multirow{2}{*}{0.29} \\
\hline G & $57(09)$ & $64(10.7)$ & & \\
\hline
\end{tabular}

ACS: acute coronary syndrome; CG: control group; CI: confidence interval; OR: odds ratio.

In ACS patients, there was no such association of these CD40 genetic variants nor an association of the CD40 rs11086998 polymorphism with CD40 plasma levels in both groups of study assuming the dominant model of association $\mathrm{C} / \mathrm{C}$ vs. $\mathrm{C} / \mathrm{G}+\mathrm{G} / \mathrm{G}$ (ACS: $846 \mathrm{pg} / \mathrm{mL}$ vs. 725 $\mathrm{pg} / \mathrm{mL}, p=0.318$; CG: $509 \mathrm{pg} / \mathrm{mL}$ vs. $540 \mathrm{pg} / \mathrm{mL}, p=0.195$ ) (Figure 4).

Regarding sCD40L plasma levels, patients exhibit higher levels of sCD40L compared with controls; however, this comparison did not reach statistical significance (1080 vs. 868.9 $\mathrm{pg} / \mathrm{mL}, p=0.16$ ). According to ACS diagnosis and sCD40L stratified by gender, female patients with STEMI had higher levels compared with UA female patients (1489.5 vs. 533.8 $\mathrm{pg} / \mathrm{mL}, p=0.026)$. UA male patients presented higher levels of sCD40L compared to UA female patients (1714.3 vs. $533.8 \mathrm{pg} / \mathrm{mL}, p=0.016$, Figure 5). When pooled, the levels of sCD40L were similar by ACS diagnosis $(p=0.75$, data not shown).

Medication did not have a significant effect on plasma sCD40L in the ACS group when introduced in the multiregression model $(\beta: 0.003, p=0.98)$; i.e., differences between patients with ACS of sCD40L are not driven by medication.
We analyzed the association between genetic variants on CD40L and plasma levels of sCD40L in both groups of study without any evidence of association.

Finally, we observed a positive linear correlation between sCD40L and IFN- $\gamma$ in ACS patients $(r=0.505, p=0.003)$ (Figure 6).

\section{Discussion}

CD40 and CD40L have important functions that comprise cellular activation and production of proinflammatory cytokines. This promotes inflammation that can accelerate atherosclerosis processes and have a functional role in the development of cardiovascular diseases [8]. Therefore, genetic variants have been of interest for their relationship with the increased risk of cardiovascular diseases [14, 15].

The SNP on the CD40 gene, rs1883832, has been associated with ACS in the presence of the major $\mathrm{C}$ allele and has been related with decreased levels of sCD40 in the presence of the minor $\mathrm{T}$ allele and risk of cardiovascular diseases $[31,32]$. In a western Mexican cohort, this was not identified as a genetic risk factor to rheumatoid arthritis (RA) [25]. This polymorphism has been associated with diminished mRNA expression of CD40 [21, 22]; rs1883832 has been found in high LD with rs4810485, the latter being significantly associated with coronary artery lesion formation in a Taiwanese population [33]. Given the localization on region $5^{\prime}$ UTR and intron 1 of these SNPs, respectively, they have been associated with changes in mRNA expression because they are in

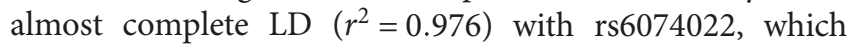
resides within the CD40 promoter and affects levels of mRNA [20]. Otherwise, to the best of our knowledge, rs11086998 has not been associated as a genetic risk factor in cardiovascular diseases, but it is known to be related in the modulation of the levels of proinflammatory cytokines, TNF $\alpha$ and IL-6, because it resides on exon 9 encoding the intracellular domain protein implicated in signaling pathways of CD40 [34].

These polymorphisms have been previously identified as genetic markers of cardiovascular disease mainly in the Chinese and European population: $\mathrm{Li}$ et al. studied 160 ACS patients and 92 controls in a Chinese Han population [12]; Tian et al. analyzed 248 ACS patients and 206 controls in a Chinese population [13]; Wang et al. reported the analysis in 474 patients with coronary atherosclerotic plaques and 225 controls in a Chinese population [14]; and GarcíaBermúdez et al. analyzed 290 patients with rheumatoid arthritis (RA) with cardiovascular events and 1285 patients with RA but without cardiovascular events [17]. However, in our study, we cannot support this association. This could be explained by genetic differences among populations [35]. The three variants on CD40 were found in LD which means they need to be analyzed together at least in our population. In this context, we found that the haplotype block rs1883832 (C)-rs4810485 (T)-rs11086998 (C) is associated with a decreased risk of ACS (OR: 0.22; $p=0.0001)$ and it may be a protective factor of ACS in the western Mexican population, although it cannot be ruled out that other 
TABLE 3: Genotype and allele frequency of the CD40L rs3092952 and rs3092920 in the ACS and CG by gender.

\begin{tabular}{|c|c|c|c|c|c|c|}
\hline & $\begin{array}{c}\text { ACS } \\
n(\%)\end{array}$ & $\begin{array}{c}\text { CG } \\
n(\%)\end{array}$ & OR (CI 95\%) & $p$ & & $p^{\mathrm{a}}$ \\
\hline rs3092952 & & & & & MAF & \\
\hline Allele & & & Male & & & \\
\hline A & $115(46.7)$ & 89 (54.9) & & & & \\
\hline G & $131(53.3)$ & $73(45.1)$ & $1.39(0.93-2.07)$ & 0.11 & 0.50 & \\
\hline Allele & & & Female & & & 0.73 \\
\hline A & $68(45.9)$ & $154(50)$ & & & & \\
\hline G & $80(54.1)$ & $154(50)$ & $1.18(0.79-1.74)$ & 0.42 & 0.51 & \\
\hline Genotype & & & Female & & & \\
\hline $\mathrm{A} / \mathrm{A}$ & $18(24.3)$ & $37(24)$ & 1 & - & & \\
\hline $\mathrm{A} / \mathrm{G}$ & $32(43.2)$ & $80(52)$ & $0.82(0.41-1.65)$ & 0.58 & - & - \\
\hline $\mathrm{G} / \mathrm{G}$ & $24(32.5)$ & $37(24)$ & $1.33(0.62-2.86)$ & 0.46 & & \\
\hline rs3092920 & & & & & MAF & \\
\hline Allele & & & Male & & & \\
\hline G & $197(80)$ & $138(85.2)$ & $143(0.94244)$ & 010 & 0.180 & \\
\hline $\mathrm{T}$ & $49(20)$ & $24(14.8)$ & $1.43(0.04-2.44)$ & 0.19 & 0.180 & \\
\hline Allele & & & Female & & & 0.96 \\
\hline G & $120(81)$ & $253(82.1)$ & $0.03(0-56154)$ & 078 & 0182 & \\
\hline $\mathrm{T}$ & $28(19)$ & $55(17.9)$ & $0.93(0.56-1.54)$ & 0.18 & 0.182 & \\
\hline Genotype & & & & & & \\
\hline $\mathrm{G} / \mathrm{G}$ & $48(64.9)$ & $104(67.5)$ & 1 & - & & \\
\hline $\mathrm{G} / \mathrm{T}$ & $24(32.4)$ & $45(29.2)$ & $0.86(0.47-1.58)$ & 0.64 & - & - \\
\hline $\mathrm{T} / \mathrm{T}$ & $2(2.7)$ & $5(3.3)$ & $1.15(0.22-6.16)$ & 0.87 & & \\
\hline
\end{tabular}

ACS: acute coronary syndrome; CG: control group; CI: confidence interval; MAF: minor allele frequencies; OR: odds ratio. ${ }^{\mathrm{a}} p$ value for the allelic model between genders.

TABLE 4: CD40 haplotype distribution in the ACS patients and control group.

\begin{tabular}{lcccc}
\hline CD40 haplotype & ACS & CG & OR (CI 95\%) & $p$ \\
\hline CCG & $403(67.1)$ & $386(65.5)$ & 1 & - \\
CGG & $58(9.7)$ & $65(9.8)$ & 0.85 & 0.42 \\
CTC & $7(1.2)$ & $30(5.1)$ & 0.22 & 0.0001 \\
TTC & $132(22)$ & $108(18.3)$ & 1.17 & 0.29 \\
\hline
\end{tabular}

ACS: acute coronary syndrome; CG: control group; CI: confidence interval; corrected significance level $(\mathrm{pc})<0.016$; OR: odds ratio. Haplotype is represented by rs1883832, rs4810485, and rs11086998, respectively.

nearby SNPs in the LD block are associated with disease susceptibility [21].

The analysis of genetic variation in the $\mathrm{X}$ chromosome in diseases is an area of interest. In line with the above, in CD40L, we evaluated two SNPs located in the $5^{\prime}$ UTR (rs3092952) and $3^{\prime}$ UTR (rs3092920), they are located in different haplotype blocks and have a weak LD [36] as we corroborated. rs3092952 has been described as a functional variant by modulating the levels of sCD40L in plasma samples. While rs3092920 has been studied as a genetic marker of cardiovascular disease in RA patients and myocardial infarction, some studies did not find such association $[15,17]$, which is consistent with our results, because we did not find an association between genetic variants on the CD40L gene and risk of ACS.

Notwithstanding, other factors may increase the risk of ACS and influence adverse events [5]; our results do not show a significant role of these polymorphisms in acute coronary events. However, the cellular and molecular mechanisms that underlie the effect of these genetic variants and ACS could potentially be mediated by changes in the gene and protein expression of CD40 and CD40L.

Other studies analyzed CD40/CD40L mRNA expression levels in coronary artery disease (CHD), following the separation of peripheral blood mononuclear cells and qRT-PCR, and found significantly increased expression levels of both genes in the CHD group compared to the control group [24].

We did not find differences between CD40 mRNA levels in total peripheral blood leucocytes of ACS patients and controls subjects. This may be due to the fact that the main source of CD40 in the peripheral blood is B cells [21]. Field et al. did not find an association between the genotype and CD40 mRNA expression measured in whole blood; however, the $\mathrm{C} / \mathrm{C}$ rs1883832 genotype was associated with higher CD40 expression in B cells [21]. We suggest that further studies that analyze these subpopulations need to be carried 


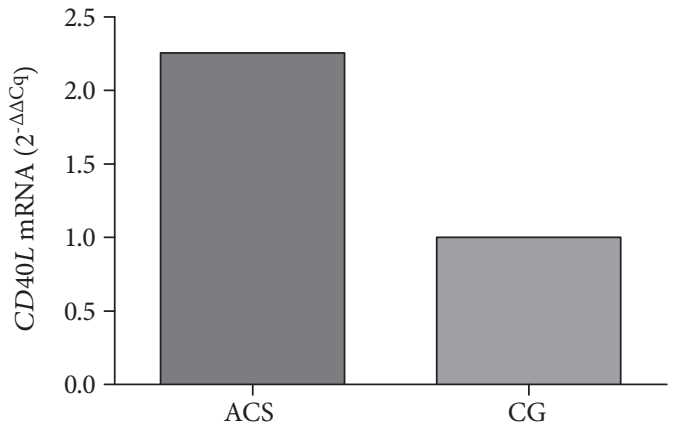

(a)

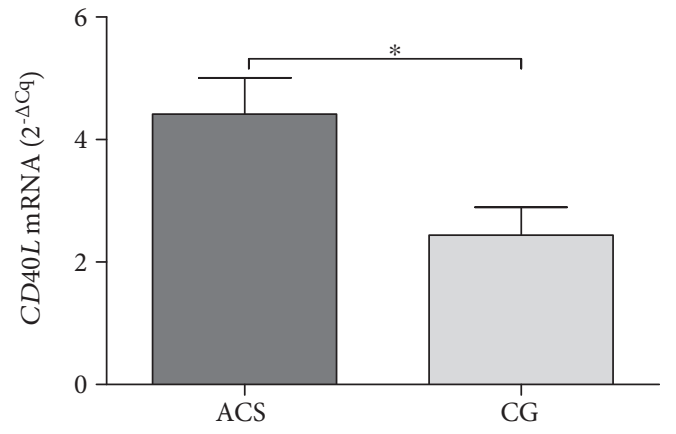

(b)

FIGURE 1: Comparison of CD40L mRNA expression in the ACS and CG. Data were analyzed by the $2^{-\Delta \Delta C q}$ and $2^{-\Delta C q}$ methods normalized to GAPDH. CD40 mRNA expression in ACS patients $(n=42)$ and CG $(n=18)$. ACS: acute coronary syndrome; CG: control group. ${ }^{*} p<0.05$ by the Mann-Whitney $U$ test.

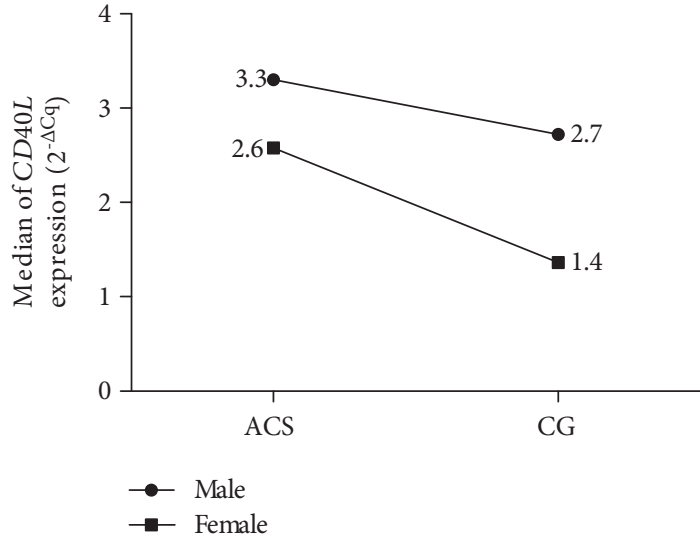

(a)

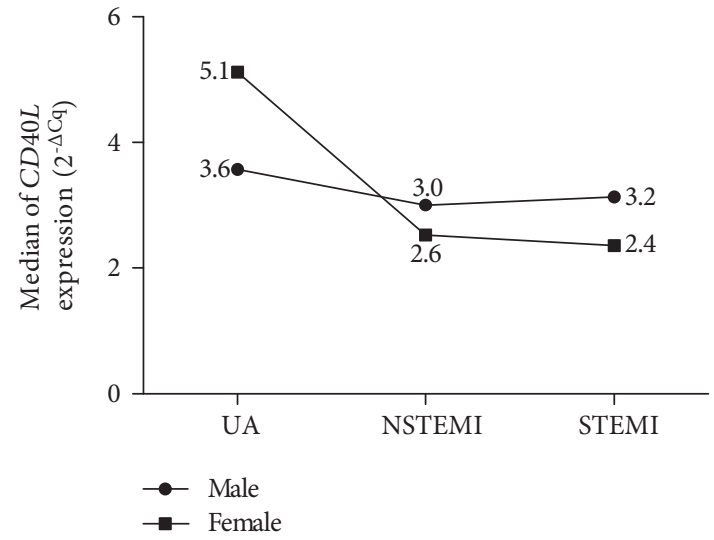

(b)

FIGURE 2: Comparison of CD4OL mRNA expression in ACS patients between genders. Data were analyzed by the $2^{-\Delta C q}$ normalized to GAPDH. CD40L mRNA expression and gender. ACS: acute coronary syndrome; CG: control group, UA: unstable angina; NSTEMI: nonST-segment elevation myocardial infarction; STEMI: ST-segment elevation myocardial infarction.

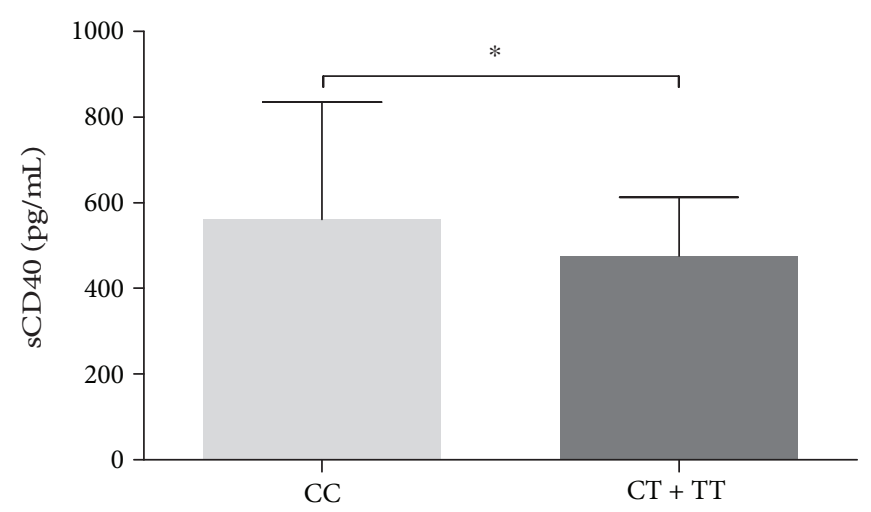

(a)

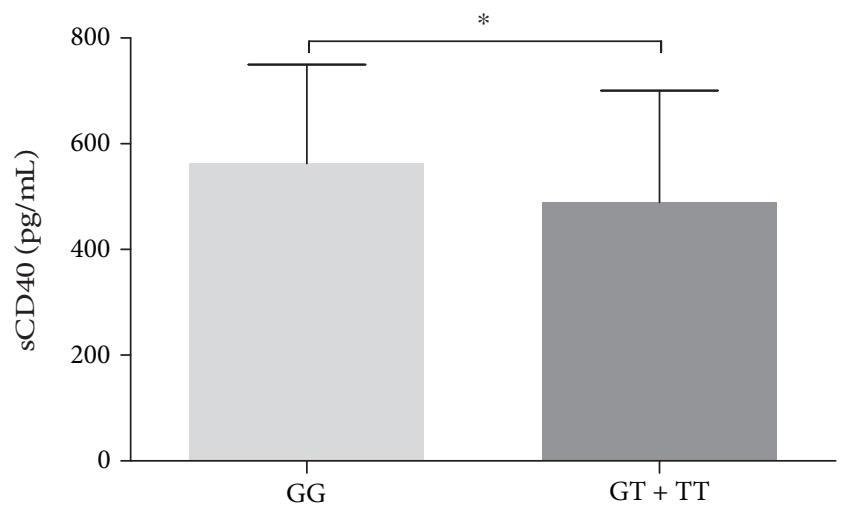

(b)

FIGURE 3: Soluble levels of CD40 are associated with rs1883832 and rs4810485 in control individuals. Association between sCD40 levels and rs1883832 (a) and rs4810485 (b) in control subjects $(n=168) .{ }^{*} p<0.05$ by the Mann-Whitney $U$ test.

out to understand the functionality of these genetic variants in CD40 mRNA gene expression.

The lack of association between genetic polymorphisms and the CD40 expression agrees with that reported by
Jacobson et al. [22], since they suggest that the rs1883832 genetic variant, which is in high linkage disequilibrium to rs4810485 ( $\mathrm{DL}=0.83$ ), acts at the level of translation rather than transcription, exerting its effects as a functional 


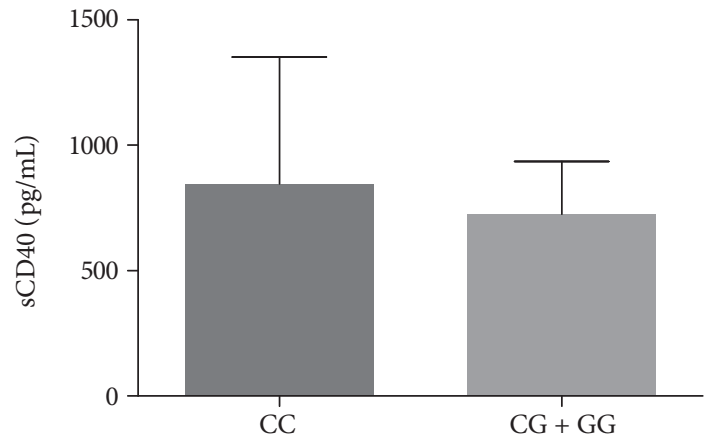

(a)

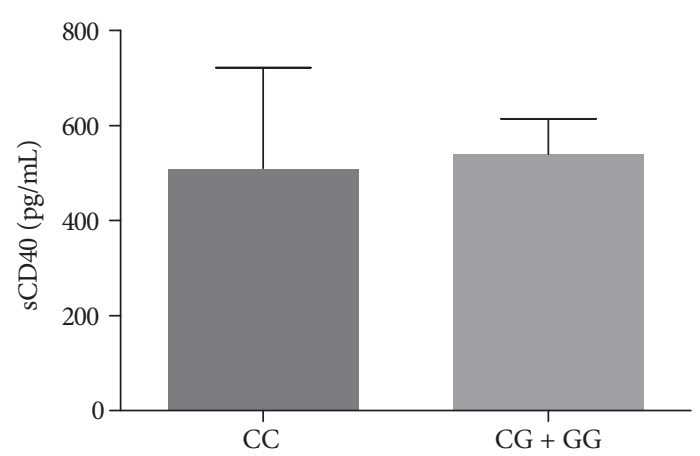

(b)

FIGURE 4: Soluble levels of CD40 and rs11086998 in ACS patients and control individuals. Association between sCD40 levels and rs11086998 in ACS patients (a) and in control subjects (b) ( $n=168$, for each group). ${ }^{*} p<0.05$ by the Mann-Whitney $U$ test.

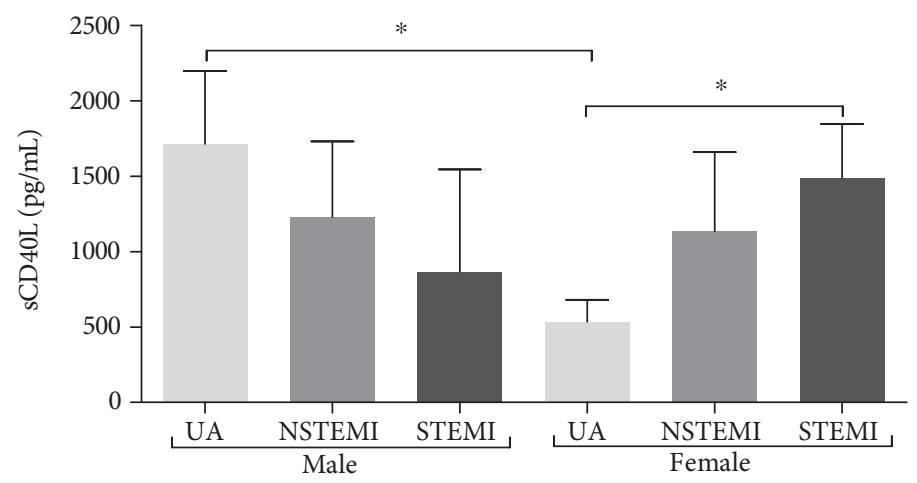

FIGURE 5: Soluble levels of CD40L in ACS patients between genders. Association between sCD40L between males $(n=136)$ and females $(n=32)$ in ACS patients $(n=168) .{ }^{*} p<0.05$ by the Mann-Whitney $U$ test.

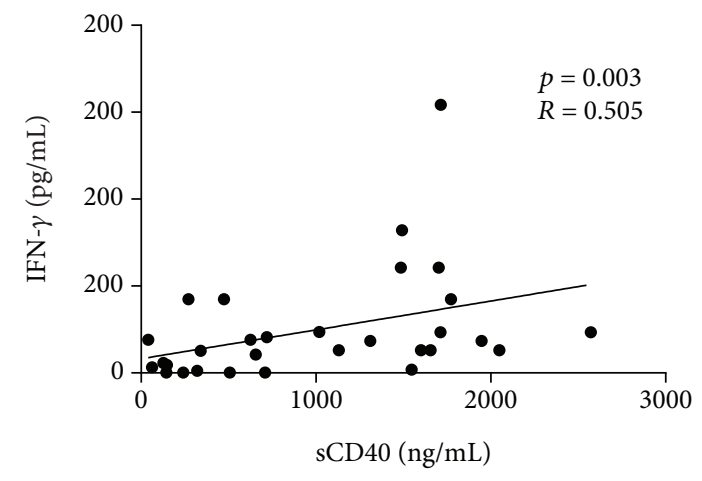

FIGURE 6: Correlation between sCD40L and IFN- $\gamma$ in ACS patients. Correlation was analyzed with Spearman's correlation coefficient $(r)$ and $p$ value.

polymorphism in the etiology of disease through the regulation of efficiency of translation, in the absence of any genotype effect on mRNA levels. In the same line, the rs4810485 SNP was associated with CD40 protein expression on $\mathrm{CD} 14^{+}$monocytes and $\mathrm{CD} 19^{+} \mathrm{B}$ cells from lupus erythematosus patients assessed by flow cytometry [18]. Other studies have reported increased levels of sCD40 in subjects who carry the minor allele of rs1883832 and rs4810485 polymorphisms $[25,32]$.
We observed increased levels of sCD40 in plasma samples from acute coronary patients compared to the control group $(p>0.001)$. During inflammatory processes, sCD40 has biological properties described as immunomodulatory, because it inhibits immune responses [37]. The proinflammatory systemic response has a key role in the pathophysiology of this syndrome contributing to the cardiovascular outcome; in this sense, sCD40 represents a potential element by which the CD40-CD40L system can be modulated through its antagonistic effect against CD40 activation [38].

The clinical entities of ACS did not differ in plasma sCD40 levels $(p=0.44)$ even when they have different outcomes, and sCD40 is involved in several stages of development of ACS [39]. We highlight that our results need to be taken with caution since clopidogrel explained $25 \%$ of the total variability of CD40 plasma levels in ACS and increased concentrations of blood lipids appeared as significant predictors of sCD40 levels (denoted by linear regression, $p=0.027$ ). Decreased cholesterol synthesis by pharmacological therapy used in ACS patients has been associated with increased levels of sCD40 [40]. It is important to clarify that clopidogrel is one of the drugs that constitute the cornerstone in the treatment scheme of these patients, so this is an intervening variable that should be considered in future studies. Unfortunately, the number of patients that is not treated with clopidogrel is quite small hampering robust comparisons. 
Pharmacological therapy can have an effect on plasma levels of CD40 [41], a circumstance that would explain the lack of association between the polymorphisms on CD40 locus and the soluble levels of plasma samples in ACS patients. An argument in favor of this is the findings in the control group; we found that homozygous carriers of the major allele of rs1883832 and rs4810485 had significantly higher levels of sCD40 (Figure 3).

Patients with ACS had increased level of CD40L mRNA compared to controls. Importantly, elevated levels of CD40L mRNA expression on $\mathrm{T}$ lymphocytes are related to acute coronary events [42]. Although we did not find significant differences between ACS and CG in SCD40L plasma levels, we observed that patients had higher levels of sCD40L and a positive linear correlation between sCD40L and IFN- $\gamma$ was found (Figure 6); thus, it may be speculated that acute coronary event patients have enhanced $\mathrm{T}$ cell responses promoting endothelial dysfunction and systemic inflammation $[43,44]$.

A previous report has concluded that CD40L mRNA expression can be affected by gender [20]; in addition, a decreased level of CD40L mRNA and increase SCD40L concentrations could reflect greater platelet activity that supports CD40L function in the immunopathology of ACS [7]. Accordingly, we only found a higher plasma sCD40L concentration in ACS patients. By clinical entities, females with UA had a lower concentration than those with STEMI; the opposite effect was seen in males. Noteworthy, the three clinical spectrums of ACS have often the same symptomatology [45] and the scientific community is urged to find biomarkers that complement the diagnosis.

Moreover, it has been reported that $4 \%$ to $30 \%$ of patients with cardiovascular ischemic diseases have poor response to antiplatelet therapy labeled as "low responders" or "nonresponders" [46]; specifically, male patients have been reported to be 2.9 times more likely to be nonresponders to antiplatelet therapy than females [47] and have been linked to recurrent cardiovascular event as death due to coronary heart disease, a major nonfatal coronary event (myocardial infarction, hospitalization for unstable angina, or resuscitated cardiac arrest), or fatal ischemic stroke [48].

The persistence of platelet reactivity even with the use of antiplatelet agents has aroused interest in strategies capable of identifying subjects with a high risk of recurrent cardiovascular events after an ACS. In this regard, plasma concentration of $\mathrm{CD} 40 \mathrm{~L}$ had been associated as a marker of myocardial damage and independently predicts death and recurrent MI in patients with ACS [10]. Hence, the adjustment of the antiplatelet therapy used in ACS according to gender and risk factors could reduce the risk of adverse events and improve the outcome of patients.

Nevertheless, we have in mind that our results should be interpreted with caution and replicated because of the sample size effect under stratified analysis.

Finally, the analysis of the association of the two genetic variants of CD40L is not correlated with mRNA expression and protein concentration. Further studies are required considering modifiable factors that can change gene expression to understand the functional effect of these polymorphisms in CD40 and CD40L expression.

\section{Conclusions}

Individually analyzed, genetic variants of CD40 and CD40L are not susceptibility markers of ACS in this Mexican cohort. However, the CTC haplotype of CD40 was associated with decreased risk of ACS.

ACS patients exhibited higher CD40L mRNA relative expression than controls.

The soluble levels of CD40 were elevated in ACS patients; however, they are affected by clopidogrel therapy.

In controls, rs $1883232 \mathrm{C} / \mathrm{C}$ and $\mathrm{rs} 4810485 \mathrm{G} / \mathrm{G}$ genotype carriers showed higher soluble levels of CD40.

Female patients with STEMI had higher levels of sCD40L compared with UA. Otherwise, UA male patients presented higher levels of sCD40L compared to UA. Medication did not have a significant effect on plasma SCD40L.

These results could reflect the inflammatory process and platelet activation in ACS patients, even when they are under antiplatelet and anti-inflammatory therapy. Due to the important roles of the CD40-CD40L system in immunologic processes in the pathogenesis of ACS, longitudinal studies are required to determine if soluble levels of CD40 and CD40L could be clinically useful markers of a recurrent cardiovascular event after an ACS.

\section{Data Availability}

The data used to support the findings of this study are included in the article.

\section{Conflicts of Interest}

The authors declare that there is no conflict of interests regarding the publication of this article.

\section{Acknowledgments}

The authors greatly appreciate the participation of every person in this study. This work was partially supported by PROSNI 2017 and PROSNI 2018 granted to YV by the University of Guadalajara.

\section{References}

[1] A. Gisterå and G. K. Hansson, "The immunology of atherosclerosis," Nature Reviews Nephrology, vol. 13, no. 6, pp. 368-380, 2017, advance online publication.

[2] J. L. Anderson and D. A. Morrow, "Acute myocardial infarction," New England Journal of Medicine, vol. 376, no. 21, pp. 2053-2064, 2017.

[3] R. S. Rosenson, H. B. Brewer, and D. J. Rader, "Lipoproteins as biomarkers and therapeutic targets in the setting of acute coronary syndrome," Circulation Research, vol. 114, no. 12, pp. 1880-1889, 2014.

[4] M. K. Reriani, A. J. Flammer, A. Jama, L. O. Lerman, and A. Lerman, "Novel functional risk factors for the prediction of cardiovascular events in vulnerable patients following acute 
coronary syndrome," Circulation Journal, vol. 76, no. 4, pp. 778-783, 2012.

[5] M. Franchini, "Genetics of the acute coronary syndrome," Annals of Translational Medicine, vol. 4, no. 10, p. 192, 2016.

[6] R. Elosua, C. Lluis, and G. Lucas, "Estudio del componente genético de la cardiopatía isquémica: de los estudios de ligamiento al genotipado integral del genoma," Revista Española de Cardiología Suplementos, vol. 9, no. 2, pp. 24-38, 2009.

[7] N. A. Michel, A. Zirlik, and D. Wolf, "CD40L and its receptors in atherothrombosis-an update," Frontiers in Cardiovascular Medicine, vol. 4, p. 40, 2017.

[8] E. Lutgens, D. Lievens, L. Beckers, M. Donners, and M. Daemen, "CD40 and its ligand in atherosclerosis," Trends in Cardiovascular Medicine, vol. 17, no. 4, pp. 118-123, 2007.

[9] C. Antoniades, C. Bakogiannis, D. Tousoulis, A. S. Antonopoulos, and C. Stefanadis, "The CD40/CD40 ligand system: linking inflammation with atherothrombosis," Journal of the American College of Cardiology, vol. 54, no. 8, pp. 669-677, 2009.

[10] N. Varo, J. A. de Lemos, P. Libby et al., "Soluble CD40L: risk prediction after acute coronary syndromes," Circulation, vol. 108, no. 9, pp. 1049-1052, 2003.

[11] M. T. Rondina, J. M. Lappé, J. F. Carlquist et al., "Soluble CD40 ligand as a predictor of coronary artery disease and long-term clinical outcomes in stable patients undergoing coronary angiography," Cardiology, vol. 109, no. 3, pp. 196-201, 2008.

[12] Y. Li, C.-X. Tian, M. Wang, and Z.-E. Xia, "Correlation of CD40 gene polymorphisms with acute coronary syndrome, hypertension and diabetes," Zhonghua Yi Xue Za Zhi, vol. 87, no. 10, pp. 690-694, 2007.

[13] C. Tian, W. Qin, L. Li, W. Zheng, and F. Qiu, "A common polymorphism in CD40 Kozak sequence $(-1 \mathrm{C} / \mathrm{T})$ is associated with acute coronary syndrome," Biomedicine \& Pharmacotherapy, vol. 64, no. 3, pp. 191-194, 2010.

[14] C. Wang, J. Yan, P. Yang, R. Du, and G. Chen, "The relationship between CD40 gene polymorphism and unstable coronary atherosclerotic plaques," Clinical Cardiology, vol. 33, no. 6, pp. E55-E60, 2010.

[15] A. Mälarstig, B. Lindahl, L. Wallentin, and A. Siegbahn, "Soluble CD40L levels are regulated by the $-3459 \mathrm{~A}>\mathrm{G}$ polymorphism and predict myocardial infarction and the efficacy of antithrombotic treatment in non-ST elevation acute coronary syndrome," Arteriosclerosis, Thrombosis, and Vascular Biology, vol. 26, no. 7, pp. 1667-1673, 2006.

[16] K. P. Burdon, C. D. Langefeld, S. R. Beck et al., "Variants of the CD40 gene but not of the CD40L gene are associated with coronary artery calcification in the Diabetes Heart Study (DHS)," American Heart Journal, vol. 151, no. 3, pp. 706-711, 2006.

[17] M. García-Bermúdez, C. González-Juanatey, R. López-Mejías et al., "Study of association of CD40-CD154 gene polymorphisms with disease susceptibility and cardiovascular risk in Spanish rheumatoid arthritis patients," PLOS ONE, vol. 7, no. 11, p. e49214, 2012.

[18] V. M. Vazgiourakis, M. I. Zervou, C. Choulaki et al., "A common SNP in the CD40 region is associated with systemic lupus erythematosus and correlates with altered CD40 expression: implications for the pathogenesis," Annals of the Rheumatic Diseases, vol. 70, no. 12, pp. 2184-2190, 2011.

[19] K. S. Gandhi, F. C. McKay, M. Cox et al., "The multiple sclerosis whole blood mRNA transcriptome and genetic associations indicate dysregulation of specific $\mathrm{T}$ cell pathways in pathogenesis," Human Molecular Genetics, vol. 19, no. 11, pp. 2134-2143, 2010.

[20] M. Wagner, M. Sobczyński, M. Bilińska et al., "MS risk allele rs1883832T is associated with decreased mRNA expression of CD40," Journal of Molecular Neuroscience, vol. 56, no. 3, pp. 540-545, 2015.

[21] J. Field, F. Shahijanian, S. Schibeci et al., "The MS risk allele of CD40 is associated with reduced cell-membrane bound expression in antigen presenting cells: implications for gene function," PloS One, vol. 10, no. 6, p. e0127080, 2015.

[22] E. M. Jacobson, E. Concepcion, T. Oashi, and Y. Tomer, “A Graves' disease-associated Kozak sequence single-nucleotide polymorphism enhances the efficiency of CD40 gene translation: a case for translational pathophysiology," Endocrinology, vol. 146, no. 6, pp. 2684-2691, 2005.

[23] Z.-L. Liu, J. Hu, S.-P. Zhao, X.-Z. Xiao, and M.-S. Yang, "The CD40 rs1883832 Polymorphism Affects Sepsis Susceptibility and sCD40L Levels," BioMed Research International, vol. 2018, Article ID 7497314, 8 pages, 2018.

[24] J. Chen, J.-H. Li, S.-J. Zhao, D.-Y. Wang, W.-Z. Zhang, and W.-J. Liang, "Clinical significance of costimulatory molecules CD40/CD40L and CD134/CD134L in coronary heart disease," Medicine, vol. 96, no. 32, article e7634, 2017.

[25] I. V. Román-Fernández, D. F. Ávila-Castillo, S. Cerpa-Cruz et al., "CD40 functional gene polymorphisms and mRNA expression in rheumatoid arthritis patients from western Mexico," Genetics and Molecular Research, vol. 15, no. 4, 2016.

[26] P. Gururajan, P. Gurumurthy, P. Nayar et al., "Increased serum concentrations of soluble CD40 ligand as a prognostic marker in patients with acute coronary syndrome," Indian Journal of Clinical Biochemistry, vol. 24, no. 3, pp. 229-233, 2009.

[27] D. Engel, T. Seijkens, M. Poggi et al., "The immunobiology of CD154-CD40-TRAF interactions in atherosclerosis," Seminars in Immunology, vol. 21, no. 5, pp. 308-312, 2009.

[28] C. P. Cannon, R. G. Brindis, B. R. Chaitman et al., "2013 ACCF/AHA key data elements and definitions for measuring the clinical management and outcomes of patients with acute coronary syndromes and coronary artery disease: a report of the American College of Cardiology Foundation/American Heart Association Task Force on Clinical Data Standards (writing committee to develop acute coronary syndromes and coronary artery disease clinical data standards)," Circulation, vol. 127, no. 9, pp. 1052-1089, 2013.

[29] P. Chomczynski and N. Sacchi, "The single-step method of RNA isolation by acid guanidinium thiocyanate-phenolchloroform extraction: twenty-something years on," Nature Protocols, vol. 1, no. 2, pp. 581-585, 2006.

[30] K. J. Livak and T. D. Schmittgen, "Analysis of relative gene expression data using real-time quantitative PCR and the 2(-delta delta C(T)) method," Methods San Diego Calif, vol. 25, no. 4, pp. 402-408, 2001.

[31] M. Wang, Y. Li, W. Li, Z. Xia, and Q. Wu, “The CD40 gene polymorphism rs1883832 is associated with risk of acute coronary syndrome in a Chinese case-control study," DNA and Cell Biology, vol. 30, no. 3, pp. 173-178, 2011.

[32] J.-M. Chen, J. Guo, C. D. Wei et al., "The association of CD40 polymorphisms with CD40 serum levels and risk of systemic lupus erythematosus," BMC Genetics, vol. 16, no. 1, 2015.

[33] H.-C. Kuo, M. C. Chao, Y. W. Hsu et al., "CD40 gene polymorphisms associated with susceptibility and coronary artery 
lesions of Kawasaki disease in the Taiwanese population," The Scientific World Journal, vol. 2012, Article ID 520865, 5 pages, 2012.

[34] A. L. Peters, R. M. Plenge, R. R. Graham et al., "A novel polymorphism of the human CD40 receptor with enhanced function," Blood, vol. 112, no. 5, pp. 1863-1871, 2008.

[35] R. Rubi-Castellanos, G. Martínez-Cortés, J. Francisco MuñozValle et al., "Pre-Hispanic Mesoamerican demography approximates the present-day ancestry of Mestizos throughout the territory of Mexico," American Journal of Physical Anthropology, vol. 139, no. 3, pp. 284-294, 2009.

[36] S. Chadha, K. Miller, L. Farwell et al., "Haplotype structure of TNFRSF5-TNFSF5 (CD40-CD40L) and association analysis in systemic lupus erythematosus," European Journal of Human Genetics, vol. 13, no. 5, pp. 669-676, 2005.

[37] C. van Kooten and J. Banchereau, "CD40-CD40 ligand," Journal of Leukocyte Biology, vol. 67, no. 1, pp. 2-17, 2000.

[38] B. D. Hock, J. L. McKenzie, N. W. Patton et al., "Circulating levels and clinical significance of soluble CD40 in patients with hematologic malignancies," Cancer, vol. 106, no. 10, pp. 21482157, 2006.

[39] M. Tsuzuki, I. Morishima, T. Yoshida et al., "Inverse correlation between soluble CD40 ligand and soluble CD40 is absent in patients with unstable angina," Heart Vessels, vol. 20, no. 6, pp. 245-250, 2005.

[40] M. Luomala, H. Päivä, R. Laaksonen et al., "Plasma-soluble CD40 is related to cholesterol metabolism in patients with moderate hypercholesterolemia," Scandinavian Cardiovascular Journal, vol. 40, no. 5, pp. 280-284, 2006.

[41] T. Sexton, E. Wallace, and S. Smyth, "Anti-thrombotic effects of statins in acute coronary syndromes: at the intersection of thrombosis, inflammation, and platelet-leukocyte interactions," Current Cardiology Reviews, vol. 12, no. 4, pp. 324329, 2016.

[42] S. X. Anand, J. F. Viles-Gonzalez, J. J. Badimon, E. Cavusoglu, and J. D. Marmur, "Membrane-associated CD40L and sCD40L in atherothrombotic disease," Thrombosis and Haemostasis, vol. 90, no. 09, pp. 377-384, 2003.

[43] J. F. McDyer, T. J. Goletz, E. Thomas, C. H. June, and R. A. Seder, "CD40 ligand/CD40 stimulation regulates the production of IFN- $\gamma$ from human peripheral blood mononuclear cells in an IL-12- and/or CD28-dependent manner," Journal of Immunology, vol. 160, no. 4, pp. 1701-1707, 1998.

[44] K. Y. Stokes, L. Calahan, C. M. Hamric, J. M. Russell, and D. N. Granger, "CD40/CD40L contributes to hypercholesterolemiainduced microvascular inflammation," American Journal of Physiology-Heart and Circulatory Physiology, vol. 296, no. 3, pp. H689-H697, 2009.

[45] High-sensitivity Cardiac Troponin for the Rapid Diagnosis of Acute Coronary Syndrome in the Emergency department: A Clinical and Cost-effectiveness Evaluation, Canadian Agency for Drugs and Technologies in Health, Ottawa, 2013, CADTH Optimal Use Report, No. 2.1A.

[46] T. K. W. Ma, Y.-Y. Lam, V. P. Tan, and B. P. Yan, "Variability in response to clopidogrel: how important are pharmacogenetics and drug interactions?," Br. J. Clin. Pharmacol., vol. 72, no. 4, pp. 697-706, 2011.
[47] R. E. J. Roach, S. C. Cannegieter, and W. M. Lijfering, "Differential risks in men and women for first and recurrent venous thrombosis: the role of genes and environment," Journal of Thrombosis and Haemostasis, vol. 12, no. 10, pp. 1593-1600, 2014.

[48] G. G. Schwartz, C. M. Ballantyne, P. J. Barter et al., "Association of lipoprotein(a) with risk of recurrent ischemic events following acute coronary syndrome: analysis of the dalOutcomes randomized clinical trial," JAMA Cardiology, vol. 3, no. 2, pp. 164-168, 2018. 


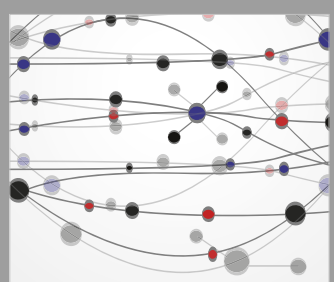

The Scientific World Journal
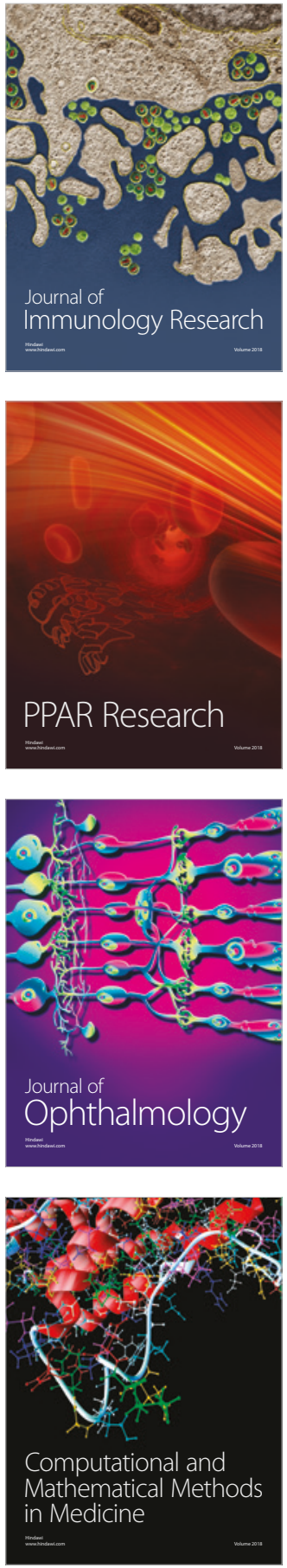

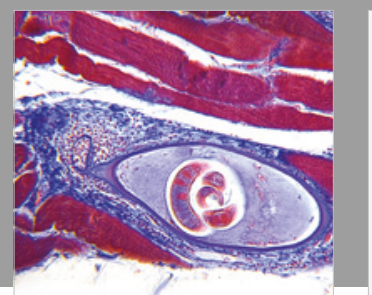

Gastroenterology Research and Practice

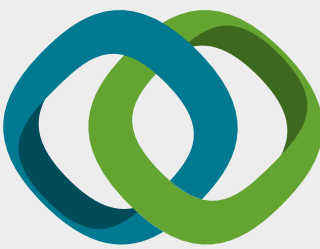

\section{Hindawi}

Submit your manuscripts at

www.hindawi.com
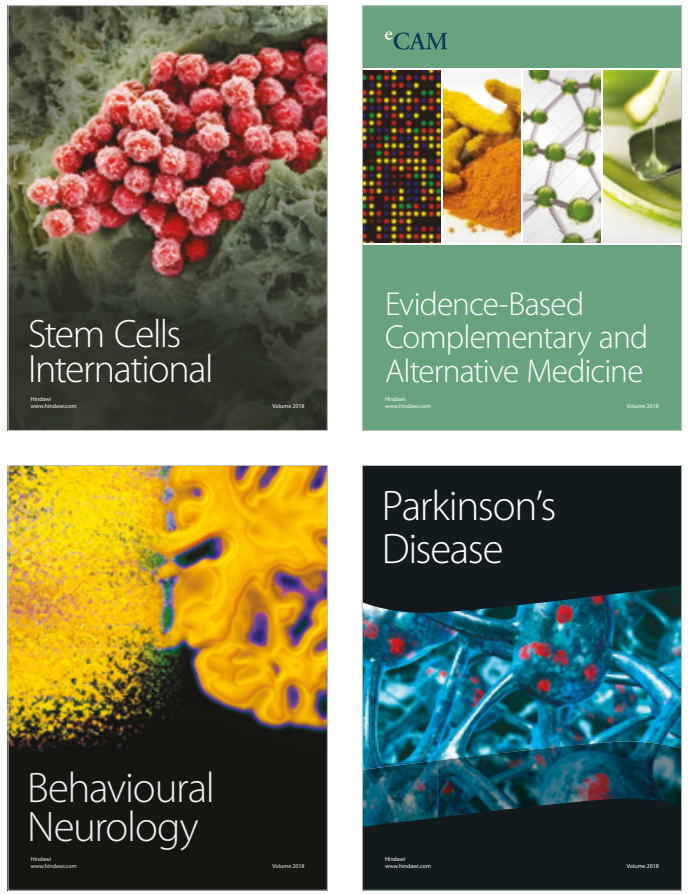

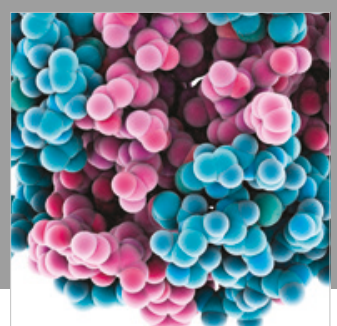

ournal of

Diabetes Research

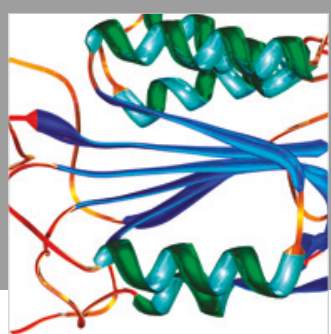

Disease Markers
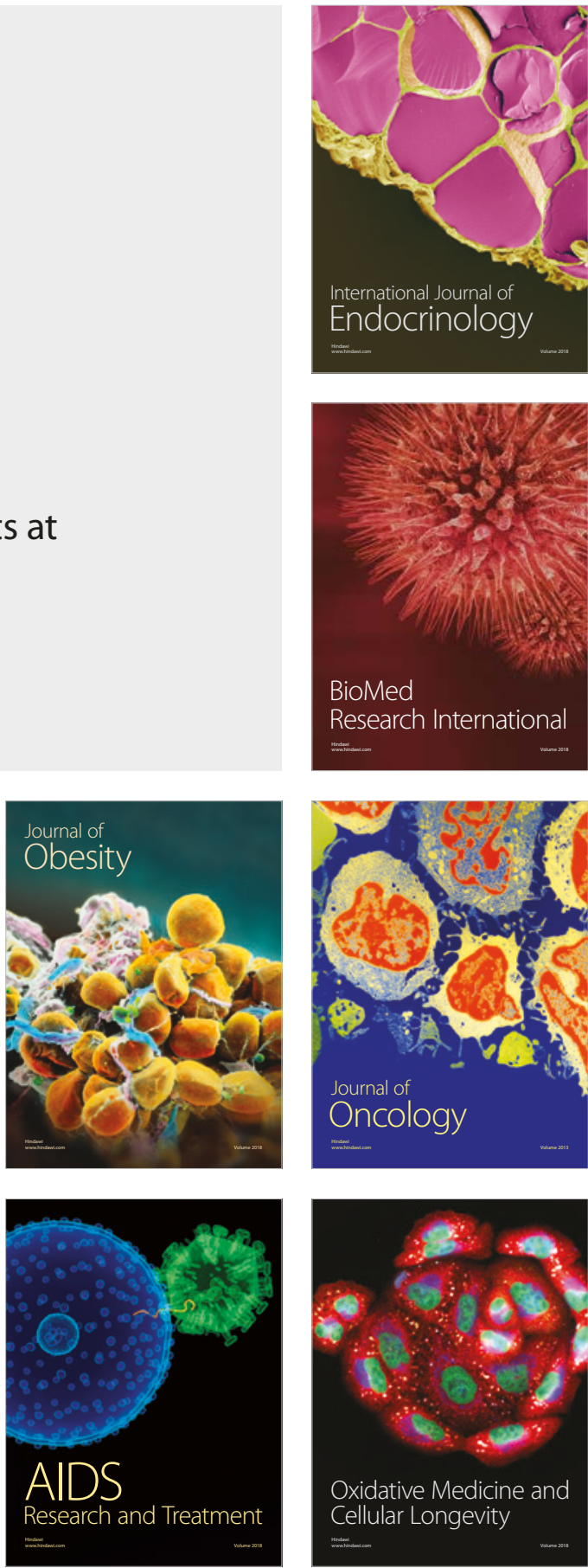